\title{
A spotlight on Raoultella ornithinolytica: A newly emerging life-threatening zoonotic pathogen
}

\author{
Wafaa A. Abd El-Ghany (i) \\ Department of Poultry Diseases, Faculty of Veterinary Medicine, Cairo University, Egypt. \\ Corresponding author: Wafaa A. Abd El-Ghany, e-mail: wafaa.ghany@yahoo.com \\ Received: 29-11-2020, Accepted: 14-01-2021, Published online: 10-02-2021
}

doi: www.doi.org/10.14202/IJOH.2021.1-5 How to cite this article: Abd El-Ghany WA (2021) A spotlight on Raoultella ornithinolytica: A newly emerging life-threatening zoonotic pathogen, Int. J. One Health, 7(1): 1-5.

\begin{abstract}
The genus Raoultella contains Raoultella ornithinolytica ( $R$. ornithinolytica), which was previously named as Klebsiella ornithinolytica. $R$. ornithinolytica is a Gram-negative bacillus that belongs to the family Enterobacteriaceae. This pathogen is normally present in aquaculture, and it has been isolated from fish, insects, and wild and domestic birds. Seafood and poultry products are incriminated as sources of $R$. ornithinolytica infection. Community-acquired human infection with $R$. ornithinolytica is common. This infection is commonly associated with sepsis, bacteremia, food poisoning, purities, and urinary and respiratory tracts' infections. Rapid acquired resistance of $R$. ornithinolytica strains is common, and it is related to the development of resistant genes. R. ornithinolytica is considered as a newly emerging life-threatening pathogen worldwide. Hence, it is very important to determine the role of animals and birds in the epidemiological situation of this pathogen as well as the detection of antibiotic resistance genes before treatment.
\end{abstract}

Keywords: Animals, antibiotic resistance, birds, human, Raoultella, susceptibility.

\section{Introduction}

Raoultella ornithinolytica $(R$. ornithinolytica) belongs to the family Enterobacteriaceae which is Gram-negative, non-motile, aerobic or facultative anaerobic, and encapsulated bacillus [1,2]. This pathogen is a member of Raoultella genus which was previously designated Klebsiella till differentiation through the phylogenetic analysis of the organism in 2001 [3-5]. The underreported incidence of $R$. ornithinolytica may be due to the inaccuracy of conventional identification methods causing uncertainties $[6,7]$ as well as the similarities with species of Klebsiella, namely, Klebsiella pneumoniae and Klebsiella oxytoca [8].

$R$. ornithinolytica is commonly found in aquatic environments [9], and it could be isolated from fish and insect species such as ticks and termites [2,10,11]. The role of $R$. ornithinolytica in animal infection has not yet been well studied. Some reports showed isolation of this pathogen from wild and domestic birds [12-17]. Moreover, R. ornithinolytica is a potent histamine producer $[18,19]$, so it became a pathogen of great importance in food products. The pathogen has also been isolated from poultry products [20] as well as seafood products $[21,22]$. It has been found that Raoultella species produced more than $1.000 \mathrm{mg}$ histamine/kg in fresh tuna [19].

It is well known that $R$. ornithinolytica is an emerging, lethal and intermittent nosocomial

Copyright: Abd El-Ghany, et al. This article is an open access article distributed under the terms of the Creative Commons Attribution 4.0 International License (http://creativecommons.org/ licenses/ by/4.0/), which permits unrestricted use, distribution, and reproduction in any medium, provided you give appropriate credit to the original author(s) and the source, provide a link to the Creative Commons license, and indicate if changes were made. The Creative Commons Public Domain Dedication waiver (http:// creativecommons.org/ publicdomain/zero/1.0/) applies to the data made available in this article, unless otherwise stated. pathogen and associated with community-acquired and/or hospital-acquired human infections [8,23,24]. The reported cases of $R$. ornithinolytica infections in humans included urinary, respiratory, and gastrointestinal tracts infections as well as wound, skin, and catheter-related blood-stream infections [25-27].

Although $R$. ornithinolytica can be treated easily, the development of resistant strains from natural environments and clinical material is common [28-30]. For example, a carbapenem-resistant $R$. ornithinolytica strain has been isolated from the urban river sediment in China [9].

Accordingly, this review article has been designed to spotlight on $R$. ornithinolytica pathogen, host susceptibility, and human infection as well as its treatment.

\section{The Pathogen}

The order Enterobacterales include genus Raoultella which is an aerobic, non-motile, Gramnegative, and encapsulated rods [3,31]. It is difficult to probably distinguish Raoultella from Klebsiella genus using conventional biochemical and phenotypic methods [32,33]. Raoultella was first classified in cluster II of Klebsiella genus and termed Klebsiella ornithinolytica [3,31,34]. Hence, ornithine decarboxylase has been used to differentiate between Raoultella and Klebsiella [28,35]. Based on sequence analysis of $16 S r R N A$, $r p o B$, gyrA, and $g y r B$ genes, the cluster II of the genus Klebsiella was renamed as a new genus Raoultella [3,36]. The genus Raoultella contains four species; $R$. ornithinolytica, Raoultella planticola, Raoultella terrigena, and Raoultella electrica $[26,37]$. The only way to achieve reliable identification of $R$. ornithinolytica and other species of Raoultella is by applying supplemental biochemical tests and/or 
using Analytical Profile Index E20 and Matrix-Assisted Laser-Desorption Ionization Time-of-Flight Mass Spectrometry [13,16,20,33,38,39]. It is oxidase-negative and catalase-positive and grows at low temperatures using sorbose as a source of carbon [3,6,31].

$R$. ornithinolytica is considered as an emerging pathogen that has some virulence factors such as capsule, and CFA/I and CFA/II colonization factors, in addition to the production of siderophores, histamine, and bacteriocins $[8,40,41]$.

\section{Host Susceptibility}

$R$ ornithinolytica is commonly present in water $[9,33]$ and has been isolated from fish [2]. The pathogen has also been found naturally in soil, vegetables, and insects as ticks and mites [6,42-45].

The isolation of $R$. ornithinolytica from animals or birds is infrequent. For instance, it has been detected in the cloacal samples of apparent healthy vulture [14] and in a case of hepatitis in ring-neck [12]. In addition, $R$. ornithinolytica has been described during a routine evaluation of aerobic enterobacteria in cloacal microbiota of birds in Argentina [16]. Mallard ducks also have been found to harbor $R$. ornithinolytica in their intestines and could act as a reservoir for this bacterium [13]. Moreover, R. ornithinolytica isolate was found in the droppings of the migratory common crane in Slovakia [15]. Recently, in Spain, $R$. ornithinolytica has been isolated from wild Canarian Egyptian vultures after feeding on fish [17].

In Thailand, previous studies demonstrated that $R$. ornithinolytica and $R$. terrigena could form a biofilm during seafood processing $[21,22,46]$. For the first time in Egypt, strains of $R$. ornithinolytica have been detected in chicken products including nuggets, strips, burgers, luncheon meats, pane, frankfurters, and minced chicken meat. Thirty-three bacterial isolates out of forty samples $(82.50 \%)$ were Enterobacteriaceae and three isolates out of 33 $(9.09 \%)$ were regarded as $R$. ornithinolytica [20].

\section{Human Infection}

The different clinical pictures of human infection with $R$. ornithinolytica are shown in Figure-1. It was documented that $R$. ornithinolytica can change histidine to histamine causing poisoning and skin flushing

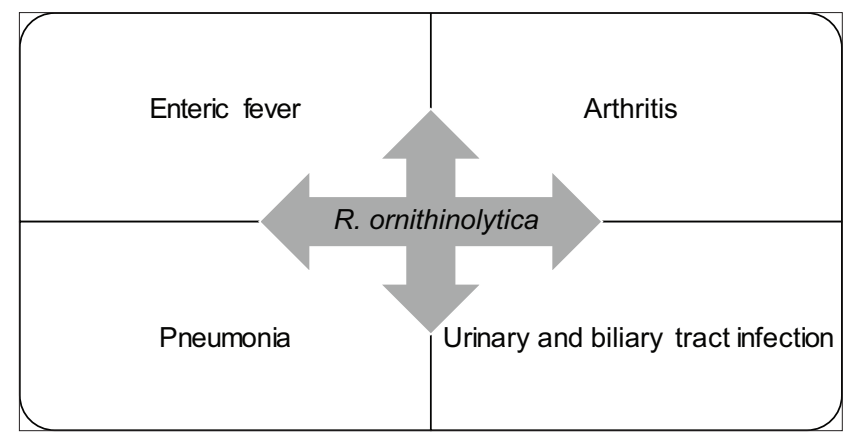

Figure-1: Clinical pictures of human infection with R. ornithinolytica. and this is called scombroid syndrome associated with fish poisoning $[25,26,40,47,48]$. As a result of increasing histamine level, patients with this syndrome showed diarrhea, vomiting, and pruritus $[11,42,49]$. Infection with $R$. ornithinolytica may induce both systemic and localized diseases' conditions especially after the abdominal surgery of cancer patients [2]. In addition, $R$. ornithinolytica was implicated in joint infection in an immunocompetent patient [1,50], and it was isolated from sepsis in patients with acute lymphocytic leukemia [51]. $R$. ornithinolytica infection is associated with food poisoning [8], sepsis [11,52-54], bacteremia [25-27,39,55-57], peritonitis [58], enteric fever [10,59], and immune deficiency conditions $[40,60]$. It has been reported that human infection with $R$. ornithinolytica results in pneumonia [61], urinary tract infection [24,49,62-64], and biliary tract disease $[7,26,39,65]$. Moreover, $R$. ornithinolytica has been isolated from patients with arthritis [66] and mandibular osteomyelitis [36]. Some reports showed $R$. ornithinolytica infection with sepsis in neonates $[11,23,53,55]$.

Most $R$. ornithinolytica patients showed recovery after antibiotic treatment, but some revealed fatal consequences, especially in immunocompromised persons $[25,56]$. Among the 69 reported cases of $R$. ornithinolytica, the mortality rate was about $20 \%$, but increased (34-44\%), especially in cases of bacteremia $[26,27,39,55-57]$.

\section{Antibiotic Treatment and Resistance}

Although $R$. ornithinolytica is not a very virulent pathogen and sensitive to antibiotics, it acquires resistance rapidly [8]. Strains of $R$. ornithinolytica were found to be sensitive to amikacin and gentamycin in USA [29], to amikacin, gentamycin, ciprofloxacin, and levofloxacin in Brazil [51], and to other carbapenemases in China [67]. The sensitivity of strains to piperacillin-tazobactam has been reported several times $[7,8,26,56,58,67]$. For 10-14 days treatment course, tigecycline, amoxicillin-clavulanic acid, levofloxacin, and cefmetazole can be used for $R$. ornithinolytica $[25,47,56]$.

To obtain good results after the treatment of $R$. ornithinolytica infection, the antimicrobial resistance of the isolates should be detected. The multidrug resistance of $R$. ornithinolytica against some antimicrobials as quinolones, cephalosporins, and carbapenems has been recorded $[68,69]$. This type of resistance is primarily associated with the development of the resistance genes [70]. The first reported case of $R$. ornithinolytica resistance was in India, where metallo- $\beta$-lactamase- 1 producing $R$. ornithinolytica was isolated from the wound in a patient with urethral injury [30]. Later, $R$. ornithinolytica antibiotic resistance has been reported in many countries like China [67], India [71] and Brazil [72].

The resistance of Raoultella strains to ampicillin $[10,56]$ that is related to the expression of 
chromosomal resistance genes encoded the production of Class A $\beta$-lactamase [28]. There was low susceptibility to $\beta$-lactam antimicrobials $[7,8,10,27,51,55,63]$. It has been recorded that $R$. ornithinolytica strains carry $b l a_{\text {DDM-1 }}$ resistant gene which is the key for the mechanism of $\beta$-lactam resistance displayed by the pathogen's strains $[28,30,73,74]$. The other carbapenem-resistant gene $\left(\right.$ bla $\left._{\mathrm{KPC}-2}\right)$ was also described in $R$. ornithinolytica isolates $[29,67]$. However, the coexistence of both genes in $R$. ornithinolytica has been recently detected [9]. A single ampicillin-resistant $R$. ornithinolytica isolate, carrying the $b l a_{\mathrm{KPC}}$ gene was detected in the droppings of a common crane [15]. Colistin-resistant genes including $m c r-1$ [43], and $m c r-8$ and its variant (mcr-8.4) were also demonstrated in $R$. ornithinolytica [69]. Some resistance of $R$. ornithinolytica to ciprofloxacin and cotrimoxazole [63] has been detected.

\section{Conclusion}

$R$. ornithinolytica is considered a newly emerging life-threatening pathogen world-wide. Hence, it is very important to determine the role of animals and birds in the epidemiological situation of this pathogen as well as the detection of antibiotic resistance genes before treatment.

\section{Author's Contributions}

WAA has collected and drafted the manuscript, formatted it, and approved the final manuscript.

\section{Acknowledgments} study.

The author did not receive any funds for this

\section{Competing Interests} interests.

The author declares that she has no competing

\section{Publisher's Note}

Veterinary World (Publisher of International Journal of One Health) remains neutral with regard to jurisdictional claims in published institutional affiliation.

\section{References}

1. Beye, M., Hasni, I., Seng, P., Michelle, C., La Scola, B., Raoult, D. and Fournier, P. (2018) Genomic analysis of a Raoultella ornithinolytica strain causing prosthetic joint infection in an immunocompetent patient. Sci. Rep., 8: 9462.

2. Hajjar, R., Ambaraghassi, G., Sebajang, H., Schwenter, F. and Su, S.H. (2020) Raoultella ornithinolytica: Emergence and resistance. Infect. Drug Resist., 13: 1091-1104.

3. Drancourt, M., Bollet, C., Carta, A. and Rousselier, P. (2001) Phylogenetic analyses of Klebsiella species delineate Klebsiella and Raoultella gen. Nov., with description of Raoultella ornithinolytica comb. Nov., Raoultella terrigena comb. Nov. and Raoultella planticola comb. Nov. Int. J. Syst. Evol. Microbiol., 51(Pt 3): 925-932.

4. Gajdács, M., Ábrók, M., Lázár, A. and Burián, K. (2019) Comparative epidemiology and resistance trends of common urinary pathogens in a tertiary-care hospital: A 10-year surveillance study. Medicina, 55(7): 356.
5. Gajdács, M., Bátori, Z., Ábrók, M., Lázár, A. and Burián, K. (2020) Characterization of resistance in Gram-negative urinary isolates using existing and novel indicators of clinical relevance: A 10-year data analysis. Life, 10(2): 16.

6. $\quad$ Park, J.S., Hong, K.H., Lee, H.J., Choi, S.H., Song, S.H., Song, K.H. and Kim, E.C. (2011) Evaluation of three phenotypic identification systems for clinical isolates of Raoultella ornithinolytica. J. Med. Microbiol., 60(Pt 4): 492-499.

7. Ponce-Alonso, M., Rodríguez-Rojas, L., del Campo, R., Cantón, R. and Morosini, M.I. (2016) Comparison of different methods for identification of species of the genus Raoultella: Report of 11 cases of Raoultella causing bacteraemia and literature review. Clin. Microbiol. Infect., 22(3): 252-257.

8. Seng, P., Boushab, B.M., Romain, F., Gouriet, F., Bruder, N., Martin, C., Paganelli, F., Bernit, E., Le Treut, Y.P., Thomas, P., Papazian, L., Raoult, D. and Stein, A. (2016) Emerging role of Raoultella ornithinolytica in human infections: A series of cases and review of the literature. Int. J. Infect. Dis., 45(2016): 65-71.

9. Dang, B., Zhang, H., Li, Z., Ma, S. and Xu, Z. (2020) Coexistence of the bla ${ }_{\text {NDM-1 }}$-carrying plasmid pWLK$\mathrm{NDM}$ and the bla ${ }_{\mathrm{KPC}-2}$-carrying plasmid pWLK-KPC in a Raoultella ornithinolytica isolate. Sci. Rep., 10(1): 2360.

10. Morais, V.P., Daporta, M.T., Bao, A.F., Campello, M.G. and Andres, G.Q. (2009) Enteric fever-like syndrome caused by Raoultella ornithinolytica (Klebsiella ornithinolytica). J. Clin. Microbiol., 47(3): 868e9.

11. Sandal, G. and Ozen, M. (2014) Fatal: Raoultella ornithinolytica sepsis and purpura fulminans in a preterm newborn. Indian J. Paediatr. Dermatol., 15(1): 24-26.

12. Gonzales-Lama, Z. and Lupiola-Gomez, P.A. (2007) Entero bactéria senhígado de Psittacus erithacus. Rev. Can. Ciên. Vet., 4(5): 11-13.

13. Murugaiyan, J., Krueger, K., Roesler, U., Weinreich, J. and Schierack, P. (2015) Assessment of species and antimicrobial resistance among Enterobacteriaceae isolated from mallard duck faeces. Environ. Monit. Assess., 187(3): 127.

14. Sala, A, Taddei, S, Santospirito, D, Sandri, C, Sandri, C., Magnone, W. and Cabassi, C.S. (2016) Antibiotic resistance in conjunctival and enteric bacterial flora in raptors housed in a zoological Garden. Vet. Med. Sci., 2(4): 239-245.

15. Hamarová, L., Repel, M., Javorský, P. and Pristaš, P. (2017) Evaluation of enteromicroflora of common crane (Grus grus) as a potential reservoir of bacterial antimicrobial resistance. Biologia, 72(9): 1098-1100

16. Marietto-Gonçalves, G.A., Lima, E.L., Nagayoshi, B.A., Tonin, A.A., Knöbl, T. and Filho, R.L.A. (2018) Raoultella ornithinolytica isolation in cloacal microbiota of Tinamus solitarius: Preliminary data. Braz. J. Poult. Sci., 20(2): 189-192.

17. Suárez-Pérez, A., Corbera, J.A., González-Martín, M., Donázar, J.A., Rosales, R.S., Morales, M. and TejedorJunco, M.T. (2020) Microorganisms resistant to antimicrobials in wild Canarian Egyptian vultures (Neophron percnopterus majorensis). Animals, 10(6): 970.

18. Tsai, Y.H., Kung, H.F., Chen, H.C., Chang, S.C., Hsu, H.H. and Wei, C.I. (2007) Determination of histamine and histamine-forming bacteria in dried milkfish (Chanos chanos) implicated in a food-borne poisoning. Food Chem., 105(3): 1289-1296.

19. Dalgaard, P. and Emborg, J. (2009) Histamine fish poisoning-new information to control a common seafood safety issue. In: Blackburn, C.W., McClure P., editors. Foodborne Pathogens: Hazards, Risk Analysis and Control. Woodhead Publishing Limited, Cambridge, UK. p1140-1160.

20. El-Shannat, S.M., El-Tawab, A.A.A. and Hassan, W.M.M. (2020) Emergence of Raoultella ornithinolytica isolated from chicken products in Alexandria, Egypt. Vet. World, 13(7): 1473-1479.

21. Hoa, B.T.Q. (2014) Assessment of Microbial Adhesion to 
Processing Surface in Shrimp Processing Plant, Biofilm Formation and Control. Kasetsart University, Bangkok, Thailand.

22. Tantasuttikul, A. and Mahakarnchanakul, W. (2019) Growth parameters and sanitizer resistance of Raoultella ornithinolytica and Raoultella terrigena isolated from seafood processing plant. Cogent Food Agric., 5(1): 1569830.

23. Abbas, A. and Ahmad, I. (2018) First report of neonatal early-onset sepsis caused by multi-drug-resistant Raoultella ornithinolytica. Infection, 46(2): 275-277.

24. De Petris, L. and Ruffini, E. (2018) Roultella ornithinolytica infection in infancy: A case of febrile urinary tract infection. CEN Case Rep., 7(2): 234-236.

25. Haruki, Y., Hagiya, H., Sakuma, A., Murase, T., Sugiyama, T. and Kondo, S. (2014) Clinical characteristics of Raoultella ornithinolytica bacteremia: A case series and literature review. J. Infect. Chemother., 20(9): 589-591.

26. Chun, S., Yun, J.W., Huh, H.J. and Lee, N.Y. (2015) Clinical characteristics of Raoultella ornithinolytica bacteremia. Infection, 43(1): 59-64.

27. Sekowska, A., Dylewska, K., Gospodarek, E. and Bogiel, T. (2015) Catheter related bloodstream infection caused by Raoultella ornithinolytica. Folia Microbiol., 60(6): 493-495.

28. Walckenaer, E., Poirel, L., Leflon-Guibout, V., Nordmann, P. and Nicolas-Chanoine, M.H. (2004) Genetic and biochemical characterization of the chromosomal class A $\beta$-lactamases of Raoultella (formerly Klebsiella) planticola and Raoultella ornithinolytica. Antimicrob. Agents Chemother., 48(1): 305-312.

29. Castanheira, M., Deshpande, L.M., DiPersio, J.R., Kang, J., Weinstein, M.P. and Jones, R.N. (2009) First descriptions of blaKPC in Raoultella spp. (R. planticola and R. ornithinolytica): Report from the SENTRY antimicrobial surveillance program. J. Clin. Microbiol., 47(12): 4129-4130.

30. Khajuria, A., Praharaj, A.K., Grover, N. and Kumar, M. (2013) First report of bla ${ }_{\mathrm{NDM}-1}$ in Raoultella ornithinolytica. Antimicrob. Agents Chemother., 57(2): 1092-1093.

31. Sakazaki, R., Tamura, K., Kosako, Y. and Yoshizaki, E. (1989) Klebsiella ornithinolytica sp. Nov., formerly known as ornithine-positive Klebsiella oxytoca. Curr. Microbiol., 18(4): 201-206.

32. Hansen, D.S., Aucken, H.M., Abiola, T. and Podschun, R. (2004) Recommended test panel for differentiation of Klebsiella species on the basis of a trilateral inter laboratory evaluation of 18 biochemical tests. J. Clin. Microbiol., 42(8): 3665-3669.

33. de Jong, E., De Jong, A.S., Smidts-van denBerg, N. and Rentenaar, R.J. (2013) Differentiation of Raoultella ornithinolytica/ planticola and Klebsiella oxytoca clinical isolates by matrix-assisted laser desorption/ionization-time of flight mass spectrometry. Diagn. Microbiol. Infect. Dis., 75(4): 431-433.

34. Mollet, C., Drancourt, M. and Raoult, D. (1997) rpoB sequence analysis as a novel basis for bacterial identification. Mol. Microbiol., 26(5): 1005-1011.

35. Boye, K. and Hansen, D.S. (2003) Sequencing of 16S rDNA of Klebsiella: Taxonomic relations within the genus and to other Enterobacteriaceae. Int. J. Med. Microbiol., 292(7-8): 495-503.

36. Lam, P.W., Tadros, M. and Fong, I.W. (2018) Mandibular osteomyelitis due to Raoultella species. JMM Case Rep., 5(3): e005140.

37. Singh, M., Kaur, I., Mundi, D.K. and Kaur, A. (2017) ENT infection caused by Raoultella ornithinolytica. Niger. J. Clin. Pract., 20(7): 914-917.

38. Seng, P., Abat, C., Rolain, J.M., Colson, P., Lagier, J.C., Gouriet, F., Fournier, P.E., Drancourt, M., La Scola, B. and Raoult, D. (2013) Identification of rare pathogenic bacteria in a clinical microbiology laboratory: Impact of matrix-assisted laser desorption ionization-time of flight mass spectrometry. J. Clin. Microbiol., 51(7): 2182-2194.
39. de Jong, E., Erkens-Hulshof, S., Velden, L.B., Voss, A., Bosboom, R., Hodiamont, C.J. and Sturm, P.D. (2014) Predominant association of Raoultella bacteremia with diseases of the biliary tract. Scand. J. Infect. Dis., 46(2): 141-143.

40. Kanki, M., Yoda, T., Tsukamoto, T. and Shibata, T (2002) Klebsiella pneumoniae produces no histamine: Raoultella planticola and Raoultella ornithinolytica strains are histamine producers. Appl. Environ. Microbiol., 68(7): 3462-3466.

41. Hulu, S.M., Al-Charrakh, A.L. and Al-Saadi, M.A.K. (2009) Isolation and characterization of Raoultella ornithinolytica from clinical specimens in Hilla city, Iraq. M. J. Babylon, 7(4): 42-47.

42. Lee, Y.C., Lin, C.M., Huang, C.Y., Huang, Y.L., Chen, H.C., Huang, T.C. and Tsai, Y.H. (2013) Determination and frying loss of histamine in striped marlin fillets implicated in a food-borne poisoning. J. Food Prot., 76(5): 860-866.

43. Luo, J., Yao, X., Lv, L. Doi, Y., Huang, X., Huang, S. and Liu, J.H. (2017) Emergence of mcr-1 in Raoultella ornithinolytica and Escherichia coli isolates from retail vegetables in China. Antimicrob. Agents Chemother., 61(10): e01139-17.

44. Ayoade, F., Mada, P.K. and Alam, M. (2018) Fat necrosis and polymicrobial wound infection caused partly by Raoultella ornithinolytica after reduction mammoplasty. BMJ Case Rep., 2018(Pt 3): doi:10.1136/bcr-2018-224234.

45. Gajdács, M. (2020) Epidemiology of Raoultella species in the context of human infections: A 10-year retrospective study in a tertiary-care hospital in Hungary. Trends Med., 20(1): 1-4.

46. Hoa, B.T.Q., Mahakarnchanakul, W., Sajjaanantakul, T. and Kitpreechavanich, V. (2015) Adhesive microflora on stainless steel coupons in seafood processing plant. J. Food Nutri. Sci., 3(1-2): 28-32.

47. Solak, Y., Gul, E.E., Atalay, H., Genc, N. and Tonbul, H.Z. (2011) A rare human infection of Raoultella ornithinolytica in a diabetic foot lesion. Ann. Saudi Med., 31(1): 93e4.

48. Kabbara, W.K., Pharm, D. and Zgheib, Y.R. (2015) Diabetic foot infection caused by Raoultella ornithinolytica. Am. J. Health Syst. Pharm., 72(24): 2147-2149.

49. Nakasone, E., Kaneshiro, R., Min, K. and Tokeshi, J. (2015) Emergence of Raoultella ornithinolytica on O'ahu: A case of community-acquired $R$. ornithinolytica urinary tract infection. Hawaii J. Med. Public Health, 74(5): 174-175.

50. Levorova, J., Machon, V., Guha, A. and Foltan, R. (2017) Septic arthritis of the temporomandibular joint caused by rare bacteria Raoultella ornithinolytica. Int. J. Oral Maxillofac. Surg., 46(1): 111-115.

51. Kaya, S.G., Bayramoglu, G., Sönmezc, M. and Köksal, I. (2015) Raoultella ornithinolytica causing fatal sepsis. Braz. J. Infect. Dis., 19(2): 230-231.

52. Sueifan, M., Moog, V., Rau, E. and Eichenauer, T. (2016) Sepsis due to Raoultella ornithinolytica in an immunocompetent patient. Anesthesiologist, 65(2): 129-133.

53. Mahabeer, Y., Singh-Moodley, A., Mackanjee, H., Perovic, O. and Mlisana, K.P. (2017) First report of neonatal bacteraemia caused by bla $a_{\mathrm{NDM}-1}$ Raoultella ornithinolytica. S. Afr. J. Infect. Dis., 32(2): 40-42.

54. Sanchez-Codez, M., Lubian-Gutierrez, M., BlancaGarcia, J.A. and Perez Aragon, C. (2019) Leclercia adecarboxylata and Raoultella ornithinolytica catheter related infection in a child with mitocondrial disease. Arch. Argent. Pediatr., 117(2): 147-149.

55. Mau, N. and Ross, L.A. (2010) Raoultella ornithinolytica bacteremia in an infant with visceral heterotaxy. Pediatr. Infect. Dis. J., 29(5): 477-478.

56. Hadano, Y., Tsukahara, M., Ito, K., Suzuki, J., Kawamura, I. and Kurai, H. (2017) Raoultella ornithinolytica bacteremia in cancer patients: Report of three cases. Int. Med., 51(22): 3193-3195.

57. Yamakawa, K., Yamagishi, Y., Miyata, K., Shimomura, Y., 
Iwata, A., Hori, T., Mikamo, H. and Okumura, A. (2016) Bacteremia caused by Raoultella ornithinolytica in two children. Pediatr. Infect. Dis. J., 35(4): 452-453.

58. Sibanda, M. (2014) Primary peritonitis caused by Raoultella ornithinolytica in a 53-year-old man. JMM Case Rep., 1(3): 1-3.

59. Sener, D., Cokhras, H., Camcioglu, Y., Akcakaya, N. and Cakir, E. (2011) Raoultella infection causing fever of unknown origin. Pediatr. Infect. Dis. J., 30(12): 1122-1123.

60. Boattini, M., Almeida, A., Cardoso, C., Cruz, C.S., Machado, C., Vesza, Z. and Moura, R. (2016) Infections on the rise: Raoultella spp., clinical and microbiological findings from a retrospective study, 2010-2014. Infect. Dis., 48(1): 87-91.

61. Van Cleve, J.R., Boucher, B.A., Smith, D.V., Croce, M.A. (2018) Ventilator associated pneumonia caused by Raoultella ornithinolytica in two immunocompetent trauma patients. Respir. Med. Case Rep., 24: 135-137.

62. Vos, B. and Laureys, M. (2009) Giant renal cyst as cause of colic obstruction. Rev. Med. Brux, 30(2): 107-109.

63. García-Lozano, T., Plá, F.J.P. and Aznar-Oroval, E. (2013) Raoultella ornithinolytica in urinary tract infections. Clinical and microbiological study of a series of 4 oncologic patients. Med. Clin., 141(3): 138-139.

64. Buyukcam, A., Liste, U., Bicakcigil, A., Kara, A. and Sancak, B. (2019) A case of Raoultella ornithinolytica urinary tract infection in a pediatric patient. J. Infect. Chemother., 25(6): 467-669.

65. Cleveland, K.O., Mazumder, S.A. and Gelfand, M.S. (2014) Association of Raoultella bacteremia with diseases of the biliary tract. Scand. J. Infect. Dis., 46(7): 541-542.

66. Venus, K., Vaithilingam, S. and Bogoch, II. (2016) Septic arthritis of the knee due to Raoultella ornithinolytica. Infection, 44(5): 691-692.

67. Zheng, B., Zhang, J. and Ji, J. (2015) Emergence of
Raoultella ornithinolytica coproducing IMP-4 and KPC-2 carbapenemases in China. Antimicrob. Agents Chemother., 59(11): 7086-7089.

68. Sekowska, A. (2017) Raoultella spp clinical significance, infections and susceptibility to antibiotics. Folia Microbiol. (Praha), 62(3): 221-227.

69. Wang, X., Wang, Y., Zhou, Y., Wang, Z., Wang, Y., Zhang, S. and Shen, Z. (2019) Emergence of colistin resistance gene mcr-8 and its variant in Raoultella ornithinolytica. Front. Microbiol., 10: 228.

70. Behzadi, P., Urbán, E. and Gajdács, M. (2020) Association between biofilm-production and antibiotic resistance in uropathogenic Escherichia coli (UPEC): An in vitro study. Diseases, 8(2): 17.

71. Bhatt, P., Tandel, K., Das, N.K. and Rathi, K.R. (2015) New Delhi metallo-betalactamase producing extensively drug-resistant Raoultella ornithinolytica isolated from drain fluid following Whipple's pancreaticoduodenectomy. Med. J. Armed Forces India, 71(Suppl 2): 609-611.

72. Carramaschi, I.N., Castro, E.A.R., Leite, J.A., Queiroz, M.M.C., Boas, M., Rangel, K. and Zahner, V. (2019) First report of Raoultella ornithinolytica carrying blaKPC-2 isolated from a dipteran muscoid collected in garbage from a public hospital in Rio de Janeiro, Brazil. Rev. Inst. Med. Trop. Sao Paulo, 61: e32.

73. Zhou, G., Guo, S., Luo, Y., Ye, L., Song, Y., Sun, G., Guo, L., Chen, Y., Han, L. and Yang, J. (2014) NDM-1producing strains, family Enterobacteriaceae, in hospital, Beijing, China. Emerg. Infect. Dis., 20(2): 340-342.

74. Sun, F., Yin, Z., Feng, J., Qiu, Y., Zhang, D., Luo, W., Yang, H., Yang, W., Wang, J., Chen, W., Xia, P. and Zhou, D. (2015) Production of plasmid-encoding NDM-1 in clinical Raoultella ornithinolytica and Leclercia adecarboxylata from China. Front. Microbiol., 6: 458. 